\title{
Risk factors and etiology of surgical site infection after radical neck dissection in patients with head and neck cancer
}

\author{
So Yeon Park ${ }^{1}$, Mi Suk Kim² ${ }^{2}$ Joong Sik Eom ${ }^{1}$, Jin Seo Lee ${ }^{1}$, and Young Soo Rho
}

${ }^{1}$ Division of Infectious Diseases, Department of Internal Medicine, Hallym University Kangdong Sacred Heart Hospital, Seoul; ${ }^{2}$ Department of Nursing, Kyungbuk College, Youngju; ${ }^{3}$ Department of Otorhinolaryngology-Head and Neck Surgery, Ilsong Memorial Institute of Head and Neck Cancer, Hallym University Kangdong Sacred Heart Hospital, Seoul, Korea

Received: April 30, 2015

Revised : May 21, 2015

Accepted: May 26, 2015

\section{Correspondence to}

Joong Sik Eom, M.D.

Division of Infectious Diseases,

Department of Internal Medicine, Hallym University Kangdong

Sacred Heart Hospital, 150

Seongan-ro, Gangdong-gu, Seoul 05355, Korea

Tel: +82-2-2224-2693

Fax: $+82-2-475-7852$

E-mail: helppl@hallym.ac.kr
Background/Aims: Surgical site infection (SSI) is a major complication after radical neck dissection (RND) in patients with head and neck cancer (HNC). We investigated the incidence, risk factors, and etiology of SSI among patients who underwent RND.

Methods: A retrospective cohort study was performed on HNC patients, excluding those with thyroid cancer, who underwent first RND at a teaching hospital between January 2006 and June 2010. Medical records were collected and analyzed to evaluate the risk factors and microbiological etiologies.

Results: A total of 370 patients underwent first RND. The overall incidence of SSI was $19.7 \%$ (73/370). Multivariate analysis showed that male sex (odds ratio [OR], 4.281; $p=0.004)$, cardiovascular diseases (OR, 1.941; $p=0.020)$, large amount of blood loss during surgery (OR, 4.213; $p=0.001$ ), and surgery lasting longer than 6 hours (OR, 4.213; $p=0.002$ ) were significantly associated with SSI. The most common causative pathogen was Staphylococcus aureus (32.6\%), and $93.2 \%$ of S. aureus isolates were methicillin-resistant. Klebsiella pneumoniae (13/92, 14.1\%), Pseudomonas aeruginosa (11/92, 12.0\%), and Enterococcus species (11/92, 12.0\%) were also frequently detected.

Conclusions: Based on our results, we predict that certain groups of patients are at high risk for SSIs after major HNC surgery. Preventive measures or close monitoring in these patients may be required to reduce the likelihood of postoperative SSIs. Furthermore, even though additional research is required, we would consider changing the prophylactic antibiotic regimens according to the causative organisms.

Keywords: Surgical site infection; Head and neck neoplasms; Microbiology

\section{INTRODUCTION}

Head and neck cancer (HNC) is the sixth most common type of cancer, accounting for an estimated 650,000 new cancer cases and 350,000 cancer deaths worldwide every year [1]. More recently, the incidence of oropharyngeal cancer in the younger population has been increasing
[2]. Surgery is the preferred treatment for HNC despite the fact that treatment of HNC is complex and involves multiple modalities. Wide resection and reconstruction as standard therapies for HNC have improved cure rates [3]. In patients with HNC, surgical site infection (SSI) has been the most frequent and significant complication, at varying rates [4-7]. The development of an SSI can cause 
prolonged hospital stays, increased health care costs, and delayed access to postoperative adjuvant therapy $[4,6,8]$. Several studies have suggested numerous risk factors for SSIs, including underlying medical conditions, smoking, alcohol use, preoperative anesthesia risk (American Society of Anesthesiologists [ASA] score), body mass in$\operatorname{dex}(\mathrm{BMI})$, presurgical tracheostomy, previous surgery, length of preoperative hospital stay, prior chemotherapy or radiotherapy, and blood loss [4-17]. However, significant discrepancies exist between the findings of these studies, and independent risk factors remain unclear.

The current study was conducted to evaluate independent risk factors associated with SSIs involving the upper respiratory or oropharyngeal mucosa in HNC. In addition, we attempted to identify the causative organisms for these infections.

\section{METHODS}

\section{Study design and patients}

We performed a retrospective cohort study to evaluate risk factors for SSIs. We reviewed the electronic medical records of patients diagnosed with HNC from January 2006 to June 2010 at Hallym University Kangdong Sacred Heart Hospital, a 70o-bed secondary-level university hospital at which the IIsong Head-and-Neck Cancer Center is located. Patients diagnosed with HNC underwent radical neck dissection involving the upper digestive or respiratory tract were included. In addition, only patients aged $>20$ years who were undergoing their first operations were included. Patients undergoing thyroid gland surgery with or without lymph node dissection were excluded. All operations were performed by surgeons with more than 5 years of experience in head and neck major oncological surgery or reconstruction. All patients included in the study received prophylactic antibiotics, and all surgical sites were disinfected with povidone iodine before incision. All patients received routine postoperative care, and surgeons or infectious disease specialists diagnosed SSIs. The data collected included age, sex, history of smoking and alcohol consumption, underlying disease, primary site of tumor and T'NM stage, ASA score, National Nosocomial Infections Surveillance System (NNIS) risk index, preoperative albumin level (mg/mL), BMI, blood loss during surgery
(mL), blood transfusion during surgery, operation time, preoperative chemotherapy, preoperative radiotherapy, duration of hospitalization before operation, causative microorganisms, and prophylactic and therapeutic antibiotics. Severity of comorbid conditions was calculated using the Charlson comorbidity index [18]. This study was approved by the Institutional Review Board of Hallym University Kangdong Sacred Heart Hospital (Seoul, South Korea) as required by the local hospital policy at the time of the study.

\section{Definitions}

According to the Centers for Disease Control and Prevention's NNIS and the criteria laid out by Horan et al. [19] and Johnson et al. [20], a SSI was defined by the occurrence of at least one of the following within 30 days of surgery: purulent drainage from the incision, spontaneous dehiscence or deliberate opening of the incisions with signs or symptoms of infection (pain, tenderness, localized swelling, redness, or heat), an isolated organism from the incision, purulent discharge from drainage, or an abscess without evidence of clinical anastomotic leakage. According to the guidelines, SSIs are classified as being either incisional or organ/space [21]. Incisional SSIs are further divided into those involving only skin and subcutaneous tissue (superficial incisional SSI) and those involving deeper soft tissue of the incision (deep incisional SSI). Organ/space SSIs involve any part of the anatomy other than incised body wall layers that were opened or manipulated during an operation.

\section{Microbiologic methods}

Microorganisms were identified by the VITEK 2 automated system (bioMerieux Inc., Hazelwood, MO, USA) in a microbiology laboratory. Antimicrobial susceptibility testing was performed using the GNI (gram-negative identification) card GPI (gram-positive identification) card in a VITEK 2 automated system according to the recommendations of the Clinical and Laboratory Standards Institute.

\section{Statistical analysis}

The chi-square or Fisher exact tests were used to compare categorical variables. Continuous variables were compared using the Student $t$ test or Mann-Whitney test. To identify independent risk factors for infection, a 
stepwise forward logistic regression analysis was used to control for the effects of confounding variables. All risk factors with a $p<0.05$ at the univariate level were included in the multivariate logistic regression.

All variables with a value of $p<0.05$ were retained in the final model. Interactions between variables were not introduced into the models. Odds ratios and their $95 \%$ confidence intervals were calculated. All tests of significance were two-tailed, and those with a $p$ value of $<0.05$ were considered significant. SPSS version 18 (SPSS Inc., Chicago, IL, USA) was used for these analyses.

\section{RESULTS}

\section{Clinical characteristics}

During the study period, a total of 370 patients with HNC were included in this study. Out of the total of 370 patients who underwent major HNC surgery, 73 (19.7\%) had SSIs. The vast majority of SSIs were in the form of deep incisional SSIs $(40 / 73,54.8 \%)$, followed by superficial incisional SSIs $(18 / 73,24.7 \%)$ and organ/space SSIs $(15 / 73,20.5 \%)$. The median time from surgery to diagnosis of SSI was 12 days (range, 1 to 29). Of the 73 SSIs, 52 SSIs (71.2\%) developed within 14 days.

The mean age ( \pm standard deviation) of the study population was $59.02 \pm 13.09$ years, and 290 patients (78.4\%) were male (Table 1). The most common underlying disease was cardiovascular disease (35.4\%, 131/370). The most common primary site of tumors was the hypopharynx (179 cases, $48.4 \%$ ), followed by the oral cavity (114 cases, $30.8 \%$ ), salivary gland (34 cases, $9.2 \%$ ), nasopharynx/sinus (29 cases, $7.8 \%$ ), neck (4 cases, 1.1\%), and other (4 cases, 1.1\%). The median duration of hospital stay was longer in patients with SSIs than in those without SSIs (78.0 [interquartile range (IQR), 40 to 119] vs. 41.0 [IQR, 24.0 to 87.5$], p<0.001$ ). Table 1 shows the demographic characteristics of the patients and the incidence of SSIs. SSIs were found to be more frequent in men than in women $(p=0.001)$, and patients who were older $(p=0.019)$, smokers $(p=0.005)$, or consumers of alcohol $(p=0.041)$ were more likely to develop SSIs than their counterparts.

\section{Risk factors for SSIs}

The association of SSIs with preoperative variables is summarized in Table 1. Univariate analysis showed that cardiovascular disease $(p=0.006)$ and neurologic disease $(p=0.022)$ were associated with SSIs.

Table 2 summarizes the association of SSIs with perioperative variables. In the univariate analysis, an ASA score $\geq 3(p=0.005)$, NNIS risk index greater than 2 $(p<0.001)$, and operation time $\geq 6$ hours $(p=0.007)$ were associated with SSIs. The amount of bleeding was greater in patients with SSIs than in those without SSIs (900 $\mathrm{mL}$ [IQR, 700 to 1,200 ] vs. $700 \mathrm{~mL}$ [IQR, 500 to 1,000$], p$ < 0.001). We determined a cutoff point for developing SSIs. Using a receiver operating characteristic curve, we determined blood loss during the operation of more than $560 \mathrm{~mL}$ as the optimal cutoff point. Moreover, the amount of blood transfusion was higher in patients with SSIs than without SSIs, but this finding was without statistical significance $(p=0.051)$.

Multivariate analysis revealed that being male, having a long operation time (over 6 hours), underlying cardiovascular disease, and blood loss during the operation of more than $560 \mathrm{~mL}$ were independent risk factors for SSIs (Table 3).

\section{Causative microorganisms}

A microbiological analysis was performed in all 73 patients with SSIs, and 60 patients showed positive culture results (Table 4). Twenty-nine patients had polymicrobial infections (29/60, 48.3\%). A total of 92 isolates from 60 patients with SSIs included 47 gram-positive organisms (51.5\%), 41 gram-negative organisms (43.5\%), and 5 Candida species (5.4\%). The most frequently isolated pathogen was Staphylococcus aureus (30/92, 32.6\%), followed by Klebsiella pneumoniae (13/92, 14.1\%), Pseudomonas aeruginosa (11/92, 12.0\%), and Enterococcus species (11/92, 2.0\%). Twenty-eight of the S. aureus isolates were methicillin-resistant S. aureus (MRSA) (93.3\%). The resistance rates to cefotaxime and ciprofloxacin among the K. pneumoniae isolates were $30.8 \%(4 / 13)$ and 53.8\% (7/13), respectively. The most common organisms associated with superficial incisional and deep incisional SSIs were S. aureus, followed by K. pneumoniae and P. aeruginosa. The distribution of pathogens showed a similar pattern in the two types of SSIs. S. aureus was the most common pathogen associated with organ/space SSIs, as expected. Enterobacter species were more commonly associated with organ/space SSIs $(4 / 8,50.0 \%)$ than with incisional SSIs. 
Table 1. Baseline and demographic characteristics and incidence of surgical site infections in patients undergoing radical neck dissection for head and neck cancer

\begin{tabular}{|c|c|c|c|c|}
\hline Characteristic & $\begin{array}{l}\text { Total patients } \\
\qquad(\mathrm{n}=370)\end{array}$ & $\begin{array}{l}\text { Patients with SSI } \\
\qquad(\mathrm{n}=73)\end{array}$ & $\begin{array}{l}\text { Patients without SSI } \\
\qquad(\mathrm{n}=297)\end{array}$ & $p$ value \\
\hline Male sex & $290(78.4)$ & $68(93.2)$ & $222(74.7)$ & 0.001 \\
\hline Age, yr & $59.02 \pm 13.09$ & $62.23 \pm 9.91$ & $58.23 \pm 13.66$ & 0.005 \\
\hline Smoking & $231(62.4)$ & $55(75 \cdot 3)$ & $176(59.3)$ & 0.011 \\
\hline Alcohol & $241(65.1)$ & $55(75 \cdot 3)$ & $186(62.6)$ & 0.041 \\
\hline Body mass index, $\mathrm{kg} / \mathrm{m}^{2}$ & $23 \cdot 31(20.65-25 \cdot 38)$ & $22.96(20.40-25.45)$ & $23.37(20.77-25.40)$ & 0.774 \\
\hline \multicolumn{5}{|l|}{ Underlying disease } \\
\hline Cardiovascular disease & $131(35 \cdot 4)$ & $36(49 \cdot 3)$ & $95(32.0)$ & 0.006 \\
\hline Chronic lung disease & $56(15 \cdot 1)$ & $10(13.7)$ & $46(15 \cdot 5)$ & 0.702 \\
\hline Diabetes mellitus & $51(13.8)$ & $14(19.2)$ & $37(12.5)$ & 0.136 \\
\hline Liver disease & $18(4.9)$ & $2(2.7)$ & $16(5 \cdot 4)$ & 0.544 \\
\hline Neurologic disease & $16(4 \cdot 3)$ & $7(9.6)$ & $9(3.0)$ & 0.022 \\
\hline Renal disease & $7(1.9)$ & $1(1.4)$ & $6(2.0)$ & 1.000 \\
\hline Charlson weighted index of comorbidity & $3(2-3)$ & $3(2-4)$ & $2(2-3)$ & 0.055 \\
\hline Primary site of tumor & & & & 0.602 \\
\hline Oral cavity & $114(30.8)$ & $21(28.8)$ & $93(31.3)$ & \\
\hline Salivary gland & $34(9.2)$ & $4(5 \cdot 5)$ & $30(10.1)$ & \\
\hline Nasal cavity/paranasal cavity & $29(7.8)$ & $5(6.8)$ & $24(8.1)$ & \\
\hline Hypopharynx & $179(48.4)$ & $41(56.2)$ & $138(46.5)$ & \\
\hline Ear & $6(1.6)$ & $2(2.7)$ & $4(1.3)$ & \\
\hline Neck & $4(1.1)$ & o & $4(1.3)$ & \\
\hline Other & $4(1.1)$ & o & $4(1.3)$ & \\
\hline Preoperative albumin level, g/dL & $3.97 \pm 0.50$ & $3.90 \pm 0.46$ & $3.99 \pm 0.50$ & 0.143 \\
\hline Preoperative hospital stay, day & $6(20-10)$ & $7(2-12)$ & $6.5(2-9.5)$ & 0.458 \\
\hline Preoperative chemotherapy & $18(4 \cdot 9)$ & $4(5 \cdot 5)$ & $14(4 \cdot 7)$ & 0.764 \\
\hline Preoperative radiotherapy & $22(5 \cdot 9)$ & $6(8.2)$ & $16(5 \cdot 4)$ & 0.359 \\
\hline
\end{tabular}

Values are presented as number (\%), mean $\pm \mathrm{SD}$, or median (interquartile range).

SSI, surgical site infection.

\section{Treatment outcomes of SSIs}

Among the patients with SSIs, nine patients (12.33\%) had bacteremia secondary to SSI. Most patients $(82.2 \%, 60 / 73)$ improved within 7 days. However, 25 patients (34.3\%) required surgical drainage, and one patient died as a result of complications of SSI. Vancomycin and piperacillin/ tazobactam were widely used. Among the patients with SSIs, 29 patients (39.7\%) received combination therapy with vancomycin plus piperacillin/tazobactam.

\section{DISCUSSION}

SSIs are one of the most common nosocomial infections that increase medical expenses [22]. Patients with SSIs tend to have increased morbidity and mortality. Major surgery for HNC frequently requires opening of the mouth floor, oropharynx, nasopharynx, or proximal esophagus, and these areas are likely to be contaminated by organisms residing in the mucosal surfaces. The 19.7\% rate of SSIs observed in this study was consistent with the findings of another study published in South Korea [23] and previous studies [10]. However, our pa- 
Table 2. Perioperative variables associated with surgical site infections in patients undergoing radical neck dissection for head and neck cancer

\begin{tabular}{|c|c|c|c|c|}
\hline Characteristic & $\begin{array}{l}\text { Total patients } \\
\qquad(\mathrm{n}=370)\end{array}$ & $\begin{array}{l}\text { Patients with SSI } \\
\qquad(\mathrm{n}=73)\end{array}$ & $\begin{array}{l}\text { Patients without SSI } \\
\qquad(\mathrm{n}=297)\end{array}$ & $p$ value \\
\hline ASA score, $\geq 3$ & $52(14.2)$ & $16(21.9)$ & $36(12.2)$ & 0.034 \\
\hline NNIS risk index,$\geq 2$ & $49(13.2)$ & $16(21.9)$ & $33(11.1)$ & $<0.001$ \\
\hline $\begin{array}{l}\text { Prophylactic antibiotic administration } \\
\text { within } 1 \text { hour }\end{array}$ & $290(78.4)$ & $59(80.8)$ & $231(77.8)$ & 0.571 \\
\hline \multicolumn{5}{|l|}{ Prophylactic antibiotics } \\
\hline Ampicillin/sulbactam & $53(14 \cdot 3)$ & $12(1.4)$ & $41(13.8)$ & 0.565 \\
\hline 1st cephalosporin & $2(0.5)$ & $0(0.0)$ & $2(0.7)$ & 1.000 \\
\hline 2nd cephalosporin & $21(5 \cdot 7)$ & $2(2.7)$ & $19(6.4)$ & 0.394 \\
\hline 3rd cephalosporin & $212(57 \cdot 3)$ & $37(50.7)$ & $175(58.9)$ & 0.202 \\
\hline Fluoroquinolone & $17(4 \cdot 6)$ & $7(9.6)$ & $10(3.4)$ & 0.023 \\
\hline Aminoglycoside & $123(33.2)$ & $30(41.1)$ & $93(31.3)$ & 0.112 \\
\hline Clindamycin & $169(45 \cdot 7)$ & $40(54.8)$ & $129(43 \cdot 4)$ & 0.081 \\
\hline $\begin{array}{l}\text { Combination antibiotic therapy for } \\
\text { prophylaxis }\end{array}$ & $177(47.8)$ & $44(60.3)$ & $133(44.8)$ & 0.018 \\
\hline \multicolumn{5}{|l|}{ Incision route } \\
\hline Including oral cavity & $113(30.5)$ & $20(27.4)$ & $93(31.3)$ & 0.515 \\
\hline Blood transfusion, $\mathrm{mL}$ & $200(0-400)$ & $400(0-600)$ & $200(0-400)$ & 0.051 \\
\hline Blood loss during operation $\geq 560 \mathrm{~mL}$ & $218(58.9)$ & $59(80.8)$ & $159(53.5)$ & $<0.001$ \\
\hline Operation time $\geq 6$ hours & $206(82.7)$ & $70(95.9)$ & $236(79 \cdot 5)$ & 0.001 \\
\hline
\end{tabular}

Values are presented as number (\%) or median (interquartile range).

SSI, surgical site infection; ASA score, American Society of Anesthesiologist's preoperative assessment score; NNIS risk index, National Nosocomial Infections Surveillance System risk index.

tients underwent surgery using clean-contaminated procedures with opening of the upper aerodigestive tract mucosa; therefore, the infection rate of our study is relatively lower than that observed in other studies under similar conditions $[5,23]$. Male sex was a significant risk factor for SSIs in the current study. This finding is consistent with the studies by Belusic-Gobic et al. [10] and Lee et al. [23], but inconsistent with other studies that reported an association between underlying systemic disease and infection [24,25]. In this study, among underlying systemic diseases, only cardiovascular disease showed a significant association with SSIs.

We also observed a significant correlation between long operation times and SSIs. If the operation time is prolonged, there are more chances for exposure to microorganisms in the mucous area; thus, allowing for more chances for infection.

In this study, the most common pathogen associat- ed with SSIs was MRSA, followed by K. pneumoniae, P. aeruginosa, and Enterococcus species. Previous studies found similar results [5,10]. Moreover, 30.8\% and 53.8\% of $K$. pneumoniae isolates were resistant to cefotaxime and ciprofloxacin, respectively. According to common pathogens and antibiotic resistance rates, we could recommend a combination of vancomycin and anti-pseudomonal penicillin or anti-pseudomonal cephalosporin as initial empirical therapy.

All surgeries in this study were clean-contaminated and involved opening of the mucosa in the oral cavity or oropharynx or tracheobronchial tree. In a similar recent study by Lee et al. [26], early postoperative hypoalbuminemia was found to be the only independent risk factor for the development of SSI in patients undergoing major HNC surgery. Risk factors for developing SSI in colorectal surgeries known as clean-contaminated surgery were the presence of ostomy, preoperative steroids, and 
Table 3. Independent risk factors for surgical site infections in patients undergoing radical neck dissection for head and neck cancer

\begin{tabular}{lll}
\hline Risk factor & Adjusted OR (95\% CI) & $p$ value \\
\hline Male sex & $4.281(1.608-11.396)$ & 0.004 \\
Cardiovascular disease & $1.941(1.108-3.400)$ & 0.020 \\
Blood loss during operation $\geq 560 \mathrm{~mL}$ & $4.281(1.608-11.396)$ & 0.001 \\
Operation time $\geq 6$ hours & $4.213(1.227-14.469)$ & 0.002 \\
\hline
\end{tabular}

OR, odds ratio; CI, confidence interval.

Table 4. Distribution of major microorganisms causing surgical site infection $(n=92)$

\begin{tabular}{lc}
\hline Microorganism & No. $(\%)$ \\
\hline Gram-positive aerobes & $47(51.1)$ \\
\hline Staphylococcus aureus & $30(32.6)$ \\
\hline Enterococcus species & $11(12.0)$ \\
Coagulase-negative Staphylococcus & $5(5.4)$ \\
\hline Streptococcus pneumoniae & $1(1.1)$ \\
Gram-negative aerobes & $40(43.5)$ \\
\hline Klebsiella pneumoniae & $13(14.1)$ \\
\hline Pseudomonas aeruginosa & $11(12.0)$ \\
\hline Enterobacter species & $8(8.7)$ \\
\hline Acinetobacter baumannii & $5(5.4)$ \\
\hline Escherichia coli & $1(1.1)$ \\
\hline Morganella morganii & $1(1.1)$ \\
\hline Burkholderia cepacia & $1(1.1)$ \\
Candida & $5(5.4)$ \\
\hline
\end{tabular}

preoperative radiation [27]. Risk factors associated with SSI in patients undergoing clean surgery such as thyroidectomy were found to be obesity, alcohol use, and long operation time [28]. As mentioned above, the risk factors for SSI have been found to be different according to the type of surgery. In the current study, deep incisional SSI was the most common type of SSI, while superficial SSI was the most common type of SSI in clean surgery [28]. The incidence of deep incisional SSI and organ/space SSI in HNC surgery was higher than that in other types of surgery. Therefore, if SSI develops after HNC surgery, it may be necessary to conduct radiological examination early. In addition, clinicians should be aware of the implications of low postoperative albumin and consider more intensive postoperative care in $\mathrm{HNC}$ patients.
Our study has some limitations. First, because this study was of a retrospective nature, the possibility of a limitation in performing accurate comparisons should be borne in mind. This study was observational, and thus unknown risk factors might have been unequally distributed between the two groups. Second, the incidence of SSI could have been underestimated because mild cases may have been under-reported. Nevertheless, the chance of neglected data or cases is very low because all eligible patients were investigated and all patients received very careful postoperative follow-up.

In conclusion, $19.7 \%$ of our study population developed an SSI (73/370). Multivariate analysis showed that male sex, cardiovascular disease, blood loss during the operation of more than $560 \mathrm{~mL}$, and a long operation time $\geq 6$ hours were significant risk factors for SSI. Lastly, the most commonly discovered pathogen was MRSA. Based on our results, we are able to predict certain groups of patients who are at high risk of developing SSIs after major HNC surgery. Preventive measures or close monitoring in these patients may be required to reduce the likelihood of SSIs.

\section{KEY MESSAGE}

1. Of the 370 patients who underwent radical neck dissection, 73 (19.7\%) developed surgical site infection.

2. Independent risk factors for surgical site infection were male sex, cardiovascular disease, blood loss during the operation of more than $560 \mathrm{~mL}$, and a long operation time ( $\geq 6$ hours).

3. The most common pathogen was methicillin-resistant Staphylococcus aureus. 


\section{Conflict of interest}

No potential conflict of interest relevant to this article was reported.

\section{REFERENCES}

1. Jemal A, Bray F, Center MM, Ferlay J, Ward E, Forman D. Global cancer statistics. CA Cancer J Clin 2011;61:69-90.

2. Marur S, Forastiere AA. Head and neck cancer: changing epidemiology, diagnosis, and treatment. Mayo Clin Proc 2008;83:489-501.

3. Andry G, Hamoir M, Leemans CR. The evolving role of surgery in the management of head and neck tumors. Curr Opin Oncol 2005;17:241-248.

4. Cunha TF, Soares Melancia TA, Zagalo Fernandes Ribeiro $\mathrm{CM}$, et al. Risk factors for surgical site infection in cervico-facial oncological surgery. J Craniomaxillofac Surg 2012;40:443-448.

5. Hirakawa H, Hasegawa Y, Hanai N, Ozawa T, Hyodo I, Suzuki M. Surgical site infection in clean-contaminated head and neck cancer surgery: risk factors and prognosis. Eur Arch Otorhinolaryngol 2013;270:1115-1123.

6. Park SH, Choi SM, Lee DG, et al. Emergence of extended-spectrum $\beta$-lactamase-producing escherichia coli as a cause of community-onset bacteremia in South Korea: risk factors and clinical outcomes. Microb Drug Resist 2011;17:537-544.

7. Penel N, Lefebvre D, Fournier C, Sarini J, Kara A, Lefebvre JL. Risk factors for wound infection in head and neck cancer surgery: a prospective study. Head Neck 2001;23:447-455.

8. Penel N, Fournier C, Lefebvre D, Lefebvre JL. Multivariate analysis of risk factors for wound infection in head and neck squamous cell carcinoma surgery with opening of mucosa. Study of 260 surgical procedures. Oral Oncol 2005;41:294-303.

9. Lotfi CJ, Cavalcanti Rde C, Costa e Silva AM, et al. Risk factors for surgical-site infections in head and neck cancer surgery. Otolaryngol Head Neck Surg 2008;138:74-80.

10. Belusic-Gobic M, Car M, Juretic M, Cerovic R, Gobic D, Golubovic V. Risk factors for wound infection after oral cancer surgery. Oral Oncol 2007;43:77-81.

11. Delgado-Rodriguez M, Mariscal-Ortiz M, Gomez-Ortega A, et al. Alcohol consumption and the risk of nosocomial infection in general surgery. Br J Surg 2003;90:1287-1293.
12. Girod DA, McCulloch TM, Tsue TT, Weymuller EA Jr. Risk factors for complications in clean-contaminated head and neck surgical procedures. Head Neck 1995;17:713 .

13. Kumar A, Carson JL. Perioperative anemia in the elderly. Clin Geriatr Med 2008;24:641-648.

14. Malone DL, Genuit T, Tracy JK, Gannon C, Napolitano LM. Surgical site infections: reanalysis of risk factors. J Surg Res 2002;103:89-95.

15. Mangram AJ, Horan TC, Pearson ML, Silver LC, Jarvis WR. Guideline for prevention of surgical site infection, 1999: Hospital Infection Control Practices Advisory Committee. Infect Control Hosp Epidemiol 1999;20:250-278.

16. Ogihara H, Takeuchi K, Majima Y. Risk factors of postoperative infection in head and neck surgery. Auris Nasus Larynx 2009;36:457-460.

17. Swift AC. Wound sepsis, chemoprophylaxis and major head and neck surgery. Clin Otolaryngol Allied Sci 1988;13:81-83.

18. Charlson ME, Pompei P, Ales KL, MacKenzie CR. A new method of classifying prognostic comorbidity in longitudinal studies: development and validation. J Chronic Dis 1987;40:373-383.

19. Horan TC, Gaynes RP, Martone WJ, Jarvis WR, Emori TG. CDC definitions of nosocomial surgical site infections, 1992: a modification of CDC definitions of surgical wound infections. Infect Control Hosp Epidemiol 1992;13:606-608.

20. Johnson JT, Myers EN, Thearle PB, Sigler BA, Schramm VL Jr. Antimicrobial prophylaxis for contaminated head and neck surgery. Laryngoscope 1984;94:46-51.

21. Mangram AJ, Horan TC, Pearson ML, Silver LC, Jarvis WR. Guideline for prevention of surgical site infection, 1999: Centers for Disease Control and Prevention (CDC) Hospital Infection Control Practices Advisory Committee. Am J Infect Control 1999;27:97-132.

22. Roy MC, Perl TM. Basics of surgical-site infection surveillance. Infect Control Hosp Epidemiol 1997;18:659-668.

23. Lee DH, Kim SY, Nam SY, Choi SH, Choi JW, Roh JL. Risk factors of surgical site infection in patients undergoing major oncological surgery for head and neck cancer. Oral Oncol 2011;47:528-531.

24. Schwartz SR, Yueh B, Maynard C, Daley J, Henderson W, Khuri SF. Predictors of wound complications after laryngectomy: a study of over 2000 patients. Otolaryngol Head Neck Surg 2004;131:61-68. 
25. Shah JP, Haribhakti V, Loree T'R, Sutaria P. Complications of the pectoralis major myocutaneous flap in head and neck reconstruction. Am J Surg 1990;160:352-355.

26. Lee JI, Kwon M, Roh JL, et al. Postoperative hypoalbuminemia as a risk factor for surgical site infection after oral cancer surgery. Oral Dis 2015;21:178-184.

27. Konishi T, Watanabe T, Kishimoto J, Nagawa H. Elective colon and rectal surgery differ in risk factors for wound infection: results of prospective surveillance. Ann Surg 2006;244:758-763.

28. Elfenbein DM, Schneider DF, Chen H, Sippel RS. Surgical site infection after thyroidectomy: a rare but significant complication. J Surg Res 2014;190:170-176. 